\title{
In-Space Propellant Production Using Water
}

\author{
William Notardonato, ${ }^{1}$ Wesley Johnson ${ }^{1}$, Adam Swanger ${ }^{1}$, and William McQuade ${ }^{1}$ \\ NASA Kennedy Space Center, FL, 32899
}

\begin{abstract}
A new era of space exploration is being planned. Manned exploration architectures under consideration require the long term storage of cryogenic propellants in space, and larger science mission directorate payloads can be delivered using cryogenic propulsion stages. Several architecture studies have shown that in-space cryogenic propulsion depots offer benefits including lower launch costs, smaller launch vehicles, and enhanced mission flexibility. NASA is currently planning a Cryogenic Propellant Storage and Transfer (CPST) technology demonstration mission that will use existing technology to demonstrate long duration storage, acquisition, mass gauging, and transfer of liquid hydrogen in low Earth orbit. This mission will demonstrate key technologies, but the CPST architecture is not designed for optimal mission operations for a true propellant depot. This paper will consider cryogenic propellant depots that are designed for operability. The operability principles considered are reusability, commonality, designing for the unique environment of space, and use of active control systems, both thermal and fluid. After considering these operability principles, a proposed depot architecture will be presented that uses water launch and on orbit electrolysis and liquefaction. This could serve as the first true space factory. Critical technologies needed for this depot architecture, including on orbit electrolysis, zero-g liquefaction and storage, rendezvous and docking, and propellant transfer, will be discussed and a developmental path forward will be presented. Finally, use of the depot to support the NASA Science Mission Directorate exploration goals will be presented.
\end{abstract}

\section{Nomenclature}

$\Delta V \quad=$ change in velocity required to traverse between two different locations in space

$N_{\text {refills }}=$ number of times a rocket stage is refueled

$I_{s p} \quad=$ specific impulse

$g_{o} \quad=$ gravitational constant

$m_{o} \quad=$ initial vehicle mass

$m_{f} \quad=$ final vehicle mass

$Q_{\text {ref }} \quad=$ refrigeration power

$Q_{H T} \quad=$ thermal heat load

\section{Introduction}

$\mathrm{P}$ ast and current studies have shown that propellant depots in space enable sustainable exploration of the solar system. The advantages of cryogenic propellant depots in space have been discussed since early in the space program $^{2}$. Werhner Von Braun used in-space refueling as a component of his famous human exploration of space articles in Colliers magazines in the 1950's. Most early concepts called for large orbiting complexes requiring multiple launches to assemble the depot in orbit, followed by multiple resupply missions to transport large quantities of cryogens to the orbital storage complex. Depot customers then rendezvous with the station and purchase propellants in whatever quantities they need. This architecture is analogous to a terrestrial gas station; semipermanent facilities built to provide a range of refueling capabilities. The scale and complexity of these types of depots will probably prohibit their deployment, at least until launch costs are decreased significantly. Other smaller depot studies have been performed over the years. A simpler depot concept that uses a single launch to provide

${ }^{1}$ Cryogenic Engineer, Cryogenic Test Laboratory, NE-F6, Kennedy Space Center 
refueling capability to a single dedicated mission $^{3}$ is one such proposal. The Augustine committee report ${ }^{4}$ identified in-space refueling as a "significant potential benefit to the in-space transportation system beyond low-Earth orbit", and considers both models of depots as feasible. More recently, a controversial NASA study showed the total program cost of both Lunar and Mars exploration would drop significantly if the mission architecture could benefit from in-space refueling using propellant depots ${ }^{5}$. However, perceived complexity and launch costs have helped limited the development of propellant depots, and critical technology demonstrations for microgravity handling of cryogens must be achieved before these concepts will gain acceptance by NASA mission planners.

The main advantage of propellant depots is the possibility of stationing a large quantity of propellant along the path of travel. This advantage allows the reduction of initial propellant mass and the reduction (mainly in propellant tank size, but also in engine mass) and reuse of system inert mass. Since the specific impulse of a given rocket engine is fixed, one easy way to increase the "mass efficiency" of a vehicle is to refill it. Equation 1 shows the rocket equation modified for refueling a stage $\mathrm{N}$ times, by refilling the propellant tanks once you can double the delta $\mathrm{v}$ of the stage. In this context the analogy of a gas station is appropriate. By refueling a stage at a single or multiple locations, the stage can be made much smaller to achieve the same $\Delta \mathrm{V}$. However, the gas station infrastructure already exists on the Earth; it must be created beyond the Earth.

$$
\Delta V=N_{\text {refills }} I_{S P} g_{0} \ln \frac{m_{0}}{m_{f}}
$$

While many discuss the launch of liquid cryogenic fuels, few have experience handling these liquids. Because so few have the experience of dealing with cryogenic propellants, the Air Force Space Command has a significant portion of the Range Safety manual ${ }^{6}$ dedicated to them. The manual, as written, is very expensive to comply with and is also fairly untested with liquid cryogenic propellants as a part of the payload. Only a few missions have launched with hydrogen in the payload and those have all been launched as solid hydrogen (avoiding the issue of venting except as an emergency). While many upper stages have launched with both liquid hydrogen $\left(\mathrm{LH}_{2}\right)$ and liquid oxygen (LOX), those have been done by a select few companies (or the government) who have a wealth of experience handling these fluids. Additionally, the state of art storage of cryogenic upper stages is on the order of 9 hours. Allowing companies to launch water instead of cryogenic fluids will lower both their required investment in launch pad infrastructure as well as the costs in complying with Range Safety, and it will ease the handling of their payloads.

Two important issues when discussing propellant depots are how to transport the propellant to the depot, and where it originates from. Recently large amounts of water have been discovered on the lunar surface; this fact, coupled with the knowledge that oxygen can be reclaimed from the oxidized metals locked within the lunar regolith, allows for the possibility of propellant production on the Moon's surface. These commodities could then be used to supply an orbiting depot. The $\Delta \mathrm{V}$ from the lunar surface to LEO is roughly the same as from the Earth's surface, but due to the lack of atmosphere on the Moon, reusability is much more of an option due to the lack of reentry heating and therefore the need for a heat shield on the moon. Similarly, hydrogen could be taken to Mars (or extracted from the water recently found there) and combined with the oxygen extracted there to supply depots in Martian orbit, however, if a reusable tug were to be used from the Martian surface to orbit, it would need a reusable heat shield that requires minimal (or automated) inspection.

Several proposed interplanetary missions could be dramatically enhanced by the presence of depots, even in LEO. Two that are readily apparent are the proposed flagship missions: the Jupiter-Europa Mission (JEM) and Mars Sample Return.

\section{Operability}

This focuses on assesing an in-space propellant depot from an operability perspective. Key principles to consider during design are maintaining simplicity, commonality and reliability in the system, and maximizing reusability of hardware when possible. The depot should be optimized for the space environment and active control (defined later) of the propellant should consider both thermal and fluid position control. 


\section{A. Commonality}

Minimizing the number of different commodities, especially hazardous commodities, has been identified as a top priority for operability for spacecraft systems ${ }^{8}$, hence future architectures should strive to minimize the different number of fluids required on board the spacecraft. Using common fluids reduces the overall parts count of the spacecraft fluid systems and decreases mass and complexity. Fluid needs include propulsion, power, and life support. Almost all consumable needs can be met by the utilization of single fluid - water; this includes water itself as well as its eleztrolyzed hydrogen and oxygen constituents.

1. Propulsion - Liquid hydrogen and liquid oxygen are already in use for primary space propulsion applications and exhibit the highest performance of any practical chemical propellant combination. The RL-10 and J2-X upper stage engines are considered the likely choices for future exploration needs, both of which utilize $\mathrm{LH}_{2}$ and LOX. Future advanced primary propulsion requirements can also be met using hydrogen exclusively, since it can be used for nuclear thermal and solar electric propulsion as well. Additionally, spacecraft designers need to consider hydrogen and oxygen as ideal candidates for secondary propulsion (such as reaction control and orbit boosting). Advances in active and passive thermal control will enable long duration cryogenic storage, essentially making these propellants as "storable" in space as hypergols. Use of common fluids for all propulsion needs will greatly simplify spacecraft design and operations.

2. Electrical Power - Hydrogen and oxygen have been used as power reactants in fuel cell electrical power systems since the Gemini program. For example, regenerative fuel cell power will be needed to supply electrical power to lunar bases during lunar night when solar voltaicsystems are used to produce power during lunar day. Fuel cells have a higher power density than batteries and may also be used on rovers and other remote equipment. Until nuclear power and improved battery power is available, fuel cells will be part of the exploration architecture. With the use of Proton Exchange Membrane fuel cells, ultra-high purity oxygen will not be needed to prevenet cell degradation over time meaning that propellant grade $\mathrm{LOX}$ and $\mathrm{LH}_{2}$ reactants would be sufficient.

3. Life support - Life support consumables include potable water for drinking, water for cleaning, bathing and cooking, cooling water for thermal control loops, and oxygen for atmospheric revitalization. In addition, water and oxygen are also needed in portable life support systems for extravehicular activities, heat transport and rejection, and for breathing air. Until fully closed loop life support systems can be developed, there will be always be a need to resupply these critical commodities.

\section{B. Reusability}

Another key operability principle is maximum reusability of assets in space. To help minimize overall mission mass. A prime example of this concept is reusability of the upper stage propulsion system as an Earth departure stage (EDS) after reservicing by in-space consumables stations. This allows for elimination of the dry mass of the EDS from the payload mass of the upper stage and greatly increases the payload mass to be delivered. It also simplifies the servicing of the cryogenic tanks, as reservicing a cold tank does not require chill down of large quantities of mass to cold temperatures like servicing a warm tank does.

Taking this concept to the limits of practicality, mass should be distributed in the system to locations where the concept of reusability can be utilized to its maximum extent. This means that all systems necessary for operation of a depot should reside on the depot when practical. Active components of docking systems should be placed on the depot side of the interface so the spacecraft docking plates can be made simpler. This minimizes recurring costs associated with launch dry mass. If possible, active thermal control systems should remain with the depot as well, unless it is absolutely necessary to include them on the spacecraft.

\section{Design for the Environment}

The fact that space has essentially no pressure and very low temperature provides unique advantages for cryogenic storage and distribution compared to on-Earth conditions. Vehicles that are intended to operate exclusively in space should be designed to take advantage of the unique space boundary conditions, and lowering the storage temperature to increase fluid density is a feasible method of minimizing spacecraft volumes and masses.With no atmospheric backpressure to limit the venting, cryogens can be stored well below their NBP, and storage at the triple point or below is achievable. The decrease in temperature is accompanied by an increase in fluid density, allowing for greater mass of propellant to be stored in a given volume. While densification will help increase the payload mass fraction, there are operational impacts associated with implementation of densified propellants on Earth, hence to date there have been no known vehicles that have used both densified hydrogen and 
oxygen. The assumed state of normal storage is saturated liquid at $25 \mathrm{kPa}$. This storage condition increases the density of $\mathrm{LH}_{2}$ by $6 \%$ over NBP hydrogen, and $5 \%$ over the NBP of liquid oxygen. Vehicles that are intended to operate exclusively in space should be designed to take advantage of the unique space boundary conditions, and lowering the storage temperature to increase fluid density is a feasible method of minimizing spacecraft volumes and masses.

Storage tank insulation requirements in space are also different from Earth. For example, on Earth, the surroundings generally are assumed to form a warm boundary temperature of $300 \mathrm{~K}$. However, proper design of outer insulation layers and their material properties have demonstrated that warm boundary temperatures of $220 \mathrm{~K}$ are achievable in LEO 9 . This affects the cryogenic system in two ways. First, heat leak will be reduced because the temperature gradient across the insulation will be smaller. Second, the coefficient of performance of the refrigerator system will be increased due to a lower heat sink temperature. The corresponding decrease in cryocooler power will decrease the mass of the system, but will also require a more massive radiator due to the lower temperature. A mass trade would be required to determine the optimum sink temperature for the refrigeration equipment.

\section{Active Control of Propellants}

One of the keys to successful cryogenic fluid management in space is maintaining active control of the propellant. Active control means control of both the state of the cryogen (active thermal control) as well as the position of the fluid in the tank (active fluid control). The word "control" as used here means "to exercise directing influence over, to have power over". Used in this context there is no such thing as passive thermal control, therefore terming concepts for "reduced boil off" (RBO) using cryocoolers to reduce the heat load as active thermal control is a misnomer. There is no effective control if all one can do is minimize the effect of heat leak because the state of the propellant is being controlled by external forces, not the active or passive systems in the spacecraft. To have true control one must be able to regulate or govern the state of the propellant, including decreasing the bulk fluid temperature or system pressure when desired. To have true active thermal control in these cases, the ratio of the provided refrigeration power to the vessel heat leak must be greater than 1 , that is:

$$
\frac{Q_{r e f}}{Q_{H L}}>1
$$

Active thermal control has been the topic of many thermal analysis studies recently. In these cases, active thermal control is typically restricted near the point of zero boil off (ZBO), where refrigeration is provided to maintain the cryogen at steady state. Current NASA plans for in-space cryogenic demonstrations with liquid oxygen are considering refrigeration ratios slightly higher than 1 , to account for transient heating during ascent and to maintain some level of pressure control. Plans for $\mathrm{LH}_{2}$ include reduced boil off, where the refrigerator intercepts around $80 \%$ of the heat leak using a shield at an intermediate temperature. However, capabilities above simply zero boil off are possible with a focused effort to develop larger capacity staged refrigeration systems like the Claude or Brayton cycles used in hydrogen liquefiers on Earth.

Active fluid control means the spacecraft has the ability to control the position of the fluid in the tank. Used in this context, propellant settling maneuvers are considered active fluid control; but a better example would be a tank mixing pump with a spray bar. Although it depends on a passive liquid acquisition device (LAD) to provide liquid to the pump inlet, such a system would be capable of directing liquid to a desired location in order to achieve isothermal tank conditions resulting in better thermal and pressure control. However, with proper design the pump mass flow could be used to help maintain liquid position as well. Additionaly, diffusers can be used to inject the liquid axially down the tank to accumulate at the liquid acquisition device. Active fluid control can also make use of a mechanical phase separator system similar to the centrifugal pumps used on the Space Shuttle humidity separation system. Turbulators built into injectors may also be able to separate the liquid from the vapor. Finally, magnetic forces can be used, especially for LOX, to actively control the position of the fluid. These active fluid control -methods require energy input into the liquid, but sufficiently large scale refrigerators will enable their incorporation.

\section{Depot Concept}

This section describes a proposed commercial cryogenic consumables depot placed in a $500 \mathrm{~km}$ circular orbit and based on the operational principles outlined above. The architecture is based on the concept of simplifying launch operations by launching water to a propellant production and liquefaction spacecraft (PPLS) that can electrolyze the water and liquefy hydrogen and oxygen products for storage in the customer spacecraft propellant tanks. This approach minimizes the total energy needed for the hydrogen and oxygen production and liquefaction 
becasue it eliminates many intermediate energy conversion processes in favor of direct solar power to a real time electrolyzer/liquefier system.

Water storage is simple compared to $\mathrm{LOX}$ and $\mathrm{LH}_{2}$. A water launch does not need a vacuum jacket or efficient insulation systems. Water is easier to store at thermal equilibrium, especially during ground and launch phases of operations, and it is a volumetrically efficient method of hydrogen and oxygen storage, requiring 2.3 times less volume than an equivalent quantity of $\mathrm{LOX}$ and $\mathrm{LH}_{2}$. These factors lead to much higher payload mass fractions for the propellant resupply portions of the architecture. This helps minimize the repetitive launch costs that form a large fraction of the depot cost structure, making the depot more attractive from a commercial standpoint. The launch site will not require dedicated $\mathrm{LH}_{2}$ or LOX payload ground support equipment, and water is a simple payload to process.

A propellant depot that produces $\mathrm{LH}_{2}$ and $\mathrm{LOX}$ from water is by no means a new concept. Since the mid to late 1970's numerous studies have been performed exploring the potential benefits of such a resource. The earliest of these references used during the development of this paper is a study conducted by Bock and Fisher of General Dynamics/Convair ${ }^{10}$ in 1978 . Their work centered around utilizing the projected Space Shuttle's large contingency payload capacity $(12,247 \mathrm{~kg})$ to transport liquid water to an orbiting propellant processing facility consisting of an electrolyzer and liquefiers. An External Tank (ET), which was delivered to the outpost during a previous Shuttle mission, would then be filled with $\mathrm{LH}_{2}$ and $\mathrm{LOX}$ over a period of 18 months. It was determined that this process would take roughly 57 Shuttle launches at an electrolysis rate of $9,072 \mathrm{~kg}$ of water per week. Two separate scenarios were proposed to utilize the full ET. This extra propellant could be used by the Shuttle itself to place larger payloads into higher orbits; or an orbital propulsion module (OPM), consisting of a cluster of engines able to mate up to the ET, could be used to transport payloads to desired orbits. Bock and Fisher's concept was never realized due in part to the lack of a contingency payload on the actual flight vehicle ${ }^{11}$.

Electrolyzing depots were revisited in 1991 by Moran ${ }^{11}$ in support of President George H. W. Bush's Space Exploration Initiative (SEI) missions. Baselined to support one manned lunar mission per year $(200,000 \mathrm{~kg}$ total of $\mathrm{LH}_{2}$ and LOX) the concept required a dedicated Shuttle launch to put the $14,016 \mathrm{~kg}$ depot into orbit then subsequent launches to supply the station with water. Results of the study persuaded Moran to recommend abandoning the idea for use in SEI missions due to the large masses, power requirements, maintenance concerns and costs associated with design and implementation of the system. Also of concern was the fact that the Shuttle was inadequate to launch the required $32,540 \mathrm{~kg}$ of water necessary to achieve the required fill capacity and timeline; therefore a new Heavy Lift Vehicle (HLV) would have been necessary.

The turn of the century saw yet another proposal utilizing the electrolyzing depot concept, this time by a team consisting of individuals from Boeing, NASA Marshall Space Flight Center and Sverdrup Technologies ${ }^{12}$. Their effort was funded in 2000 by the Space Solar Exploratory Research and Technology Program (SERT) and consisted of two architectures in order to perform trade studies: a cryogenic production design similar to those summarized above, and a cryogenic storage-only configuration wherein the depot would act as a "gas station," requiring periodic launches of liquefied propellant to replenish its tanks. The maximum propellant manufacturing requirement was assumed to be $500,000 \mathrm{~kg}$ per year based on an all-propulsive Mars mission scenario with a projected launch timeframe of 2015 and a technology maturation rate of $40 \%$. The cryo-production architecture required seven Delta IV launches to deliver all the components to orbit while the storage-only option needed only one Delta IV Heavy launch. In the case of the cryo-production design two different strategies were examined to supply water: first, a proposed future reusable launch vehicle (RLV), requiring 15 launches per year carrying $15,876 \mathrm{~kg}$ of water per time; and second, a proposed rail-gun launch of $250-500 \mathrm{~kg}$ ice projectiles, 6-9 times per day (possible because a water payload can handle the extreme accelerations involved). In both cases, semi-autonomous orbital maneuvering vehicles (OMV) would be required to transport the water modules from the low launch orbit to the higher processing facility and back again for reuse. The study also included technology roadmaps and recommended both Shuttle and ISS missions be conducted prior to the full scale 2015 launch date to verify various fluid transfer and storage technologies in micro-gravity.

More recently, a study done by W. Notardonato ${ }^{13}$ summarized the current state of the art in active cryogenic control systems, proposed operational design strategies and presented options for future electrolyzing propellant architectures. This effort served an as a precursor to this paper.

\section{Depot System Components}

The proposed water based depot architecture incorporates three distinct spacecraft. These are the propellant production and liquefaction spacecraft, the water supply modules, and the customer spacecraft. Details of these systems are presented next. 


\section{A. Propellant Production and Liquefaction Spacecraft}

The PPLS spacecraft is the key component of the system. It is a multifunctional spacecraft that includes all command and control systems, propulsion for orbital maneuvering, attitude control, and docking operations, a remote manipulator system, power supply and distribution network, and thermal control systems. The depot spacecraft's primary purpose is to receive water from the water supply modules (WSM), electrolyze it at high pressure, and liquefy the oxygen and hydrogen output streams. The $\mathrm{LOX}$ and $\mathrm{LH}_{2}$ are then transferred to fill the empty storage tanks on the customer spacecraft. The depot has the capability to transfer liquid water, gaseous hydrogen and oxygen, and liquid hydrogen and oxygen. The depot also provides refrigeration to the customer spacecraft for propellant conditioning while they are docked. The PPLS is a multifunctional spacecraft that includes all command and control systems - propulsion for orbital maneuvering, attitude control, docking operations, a remote manipulator system, power supply and distribution network, and thermal control systems. Also, in order to maximize reusability and minimize single-use systems, all the active components for rendezvous and docking should reside on the PPLS side.

\section{B. Water Supply Modules}

The depot facility is only supplied with the propellant feedstock consisting of high purity water. This offers many advantages. As stated earlier, water storage much less complicated than $\mathrm{LOX}$ and $\mathrm{LH}_{2}$. This helps minimize the repetitive launch costs that form a large fraction of the depot cost structure, making the depot more attractive from a commercial standpoint

The design of the WSM's should be as simple as possible. They can be mass produced for economy of scale. A water supply module should have minimum capability for attitude control, communication, and power supply, and a passive docking interface with power and water connections only. Heaters will be required on orbit and the WSM tanks can be integrated into the thermal management scheme of the PPLS, serving as a thermal energy buffer.

\section{Customer Spacecraft}

A number of different types of customers could use such a PPLS depot. A typical use would be for a customer who needs a refueling depot for an upper stage that serves as an Earth departure stage for lunar or planetary missions. For this scenerio, the upper stage would arrive at the depot with its propellant tanks nearly empty but still cold. The PPLS would initiate the docking and connection of umbilical's and then begin filling the customer's propellant tanks. All customers will be required to have standard umbilical interfaces for water, gaseous oxygen and gaseous hydrogen, and liquid oxygen and liquid hydrogen transfer. Customers will have to integrate refueling specific hardware into their vechilce's systems, such as spray bars, mixing pumps, and enhanced insulation. The PPLS would control the attitude of the system while docked to maintain maximum solar power.

\section{Potential Architectures}

There are multiple potential depot architectures since no prefered architecture/location has yet been identified. Potential locations include the Earth-Moon Lagrange points L1 and L2( located roughly 322,000 km and 444,000 $\mathrm{km}$ from Earth respectively) in a fairly stable halo orbit. Such locations would provide quick access to anywhere on the surface of the moon (for lunar resource utilization). Other potential locations those generally in Low Earth Orbit (LEO) that provide easy access from the surface of the Earth or Geostationary Orbit (GEO) for servicing current or future satellites residing in that orbit. In a truly versatile depot ("gas station") infrastructure there would be stations placed at all of these locations. However, since such a robust infrastructure would require a long time and high cost to develop, the following sections investigate which locations would be preferential for initial use by proposed NASA missions.

\section{Science Missions}

The concept of operations depicted in Figure 1 show a launch using a Delta IV vehicle, capable of 25 metric ton (MT) delivery to LEO. The Delta IV upper stage is left behind cold but almost empty. Then 20 MT of water is launched on an Atlas V. The PPLS takes 6 months to process the propellants and transfer them to the Delta IV upper stage, which is then capable for a mission beyond LEO. An exploration payload (such as Mars Sample Return) is then launched on another Delta IV, which then rendezvous with the full upper stage. The upper stage then performs a Trans Martian Injection (TMI) burn. The second Delta IV upper stage is now available for refueling, pending arrival of the next WSM, and the cycle can be repeated for another planetary mission such as the Jupiter/Europa Orbiter. In this scenario the PPLS becomes the first factory in space, converting water to LOX and $\mathrm{LH}_{2}$, capable of servicing multiple customers over many years. 
Figure 2 shows the capability of a fully refueled Centaur and CPS stage. From a $500 \mathrm{~km}$ circular LEO staging orbit, the $\Delta \mathrm{V}$ requirement for TMI is roughly $3.6 \mathrm{~km} / \mathrm{s}$. Using a fully refueled Centaur allows just over $13.5 \mathrm{MT}$ to be delivered through TMI. A $4000 \mathrm{~kg}$ payload to Mars ( $3400 \mathrm{~kg}$ Curiosity type rover $+50 \mathrm{~kg}$ sample return and 550 $\mathrm{kg}$ Mars launcher) would easily fit within this mass budget. If the rover were downsized to a mass on par with the Spirt and Oppurtunity rovers (just over one metric ton) it would allow a return mass of $225 \mathrm{~kg}$ with spacecraft growth allowances. Using the PPLS to refill the Centaur tank would ensure that the tank is full and would allow for on orbit checkout of systems before committing to TMI. Using the CPS upper stage, 46 MT can be delivered through TMI. This allows a $500 \mathrm{~kg}$ return mass with a Curiosity class rover, and a $600 \mathrm{~kg}$ return mass with an Opportunity class rover.

Initial mission design for the Jupiter Europa Orbiter (JEO) indicates that over 3 years would be spent in the inner solar system performing gravitational assists to increase the velocity of the spacecraft so that it can reach Jupiter. ${ }^{7}$ This transit time cost the Galileo mission over $\$ 200 \mathrm{M}$ in mission operations costs ${ }^{7}$. The ability to refuel an upper stage on orbit dramatically reduces this transit time by allowing for a higher energy, direct transfer instead of a long, gravitational assisted approach. The TransJupiter Injection burn requires a $\Delta \mathrm{V}$ of approximately $6.6 \mathrm{~km} / \mathrm{s}$ for direct injection. Refueling a Centaur in LEO yields an allowable payload mass of $75 \mathrm{~kg}$ to study Jupiter, whereas refueling a CPS would allow a payload mass of $175 \mathrm{~kg}$.

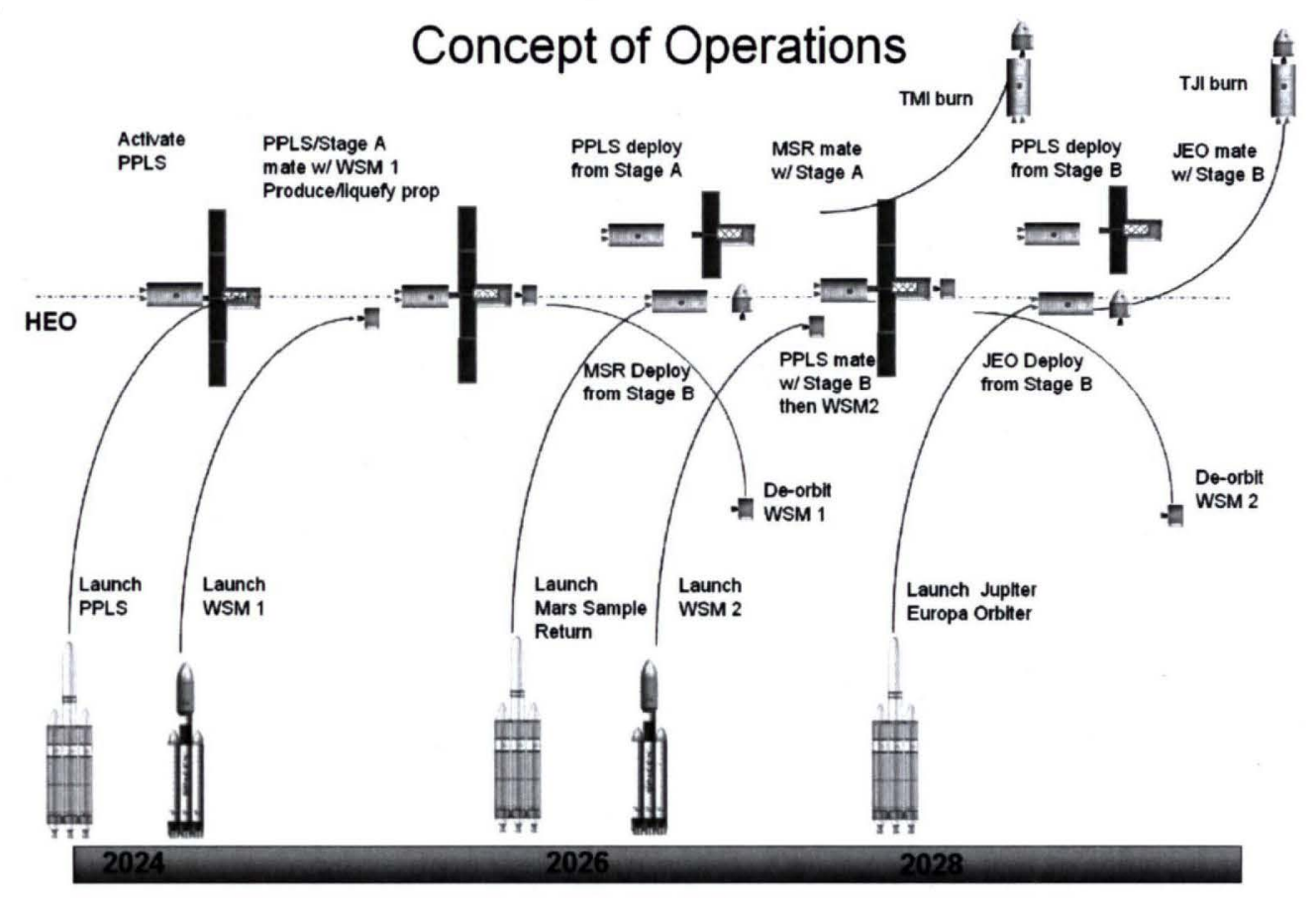

Figure 1: Concept of Operations 


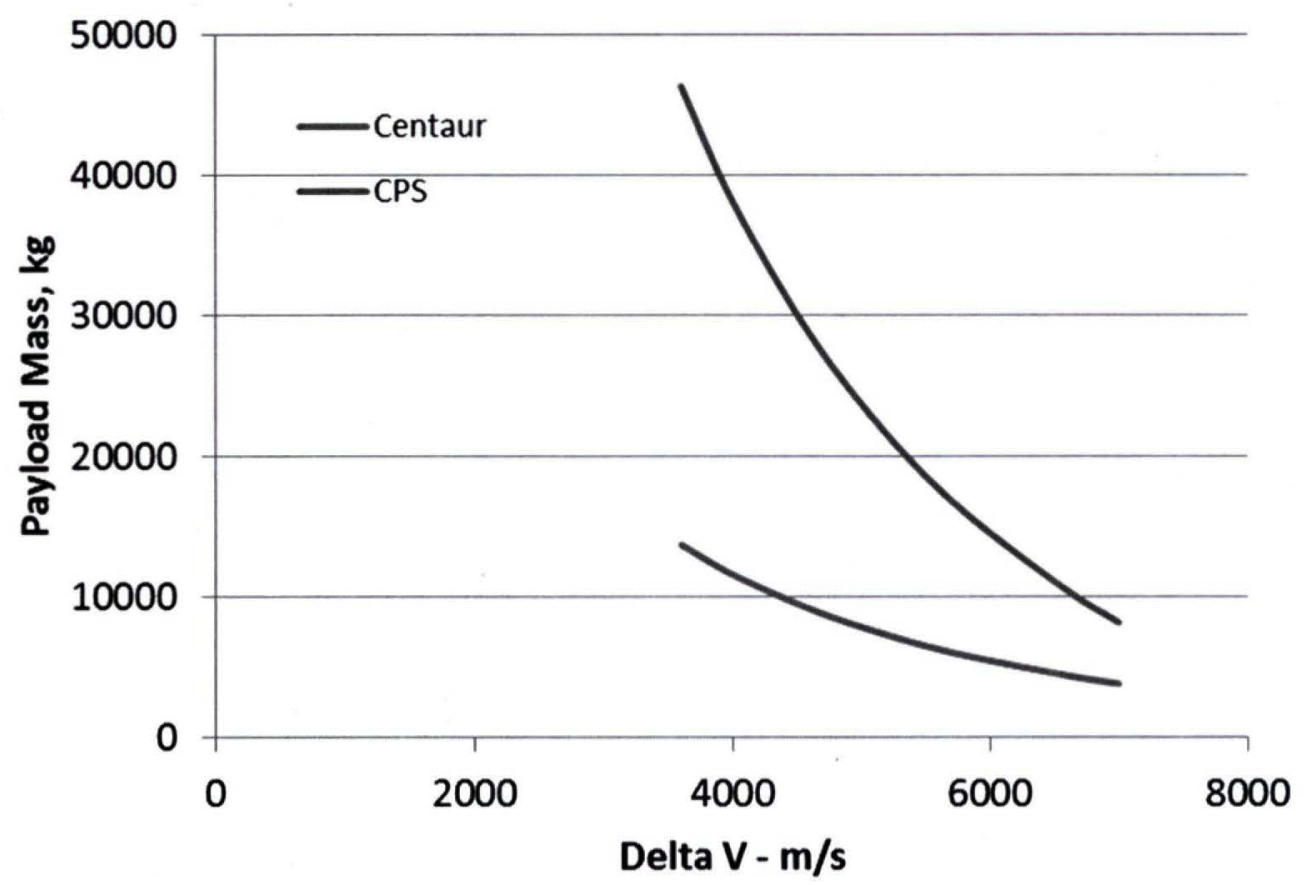

Figure 2: Payload Mass vs $\Delta V$ for a refueled Centaur and CPS

\section{L1 Depot}

Locating the PPLS at L1 (near side of the moon) instead of High Earth Orbit (HEO) or GEO would allow for easier utilization of the lunar resources. The $\Delta \mathrm{V}$ from the lunar surface to $\mathrm{L} 1$ is just over $2.5 \mathrm{~km} / \mathrm{s}$ and to LEO is roughly $2.7 \mathrm{~km} / \mathrm{s}$; whereas the $\Delta \mathrm{V}$ from the Earth surface to LEO is roughly $10 \mathrm{~km} / \mathrm{s}$, to GEO is roughly $14 \mathrm{~km} / \mathrm{s}$, and to $\mathrm{L} 1$ is roughly $14 \mathrm{~km} / \mathrm{s}$. Thus the size of the rocket to deliver a given payload is much smaller. While the advantage does not necessarily work for launching a payload from the earth, the propellant required to maintain the orbit around L1 is much less than maintaining an orbit in LEO. The trade between propellant used to make deliveries from earth (if desired) and station keeping at the L1 depot should be studied in addition to the propellant requirements over time for a Lunar orbit depot. L1 would put the spacecraft into a much higher energy orbit allowing for pushing much larger payloads beyond Earth orbit, even compared to Lunar orbit.

One advantage that a depot at Ll has is a nearly continuous view of the sun for power. Depots placed in LEO, lunar orbit or even GEO would spend a much larger time in the shadow of the body it is orbiting than at L1. This would remove the need for batteries to continue powering the liquefaction cycle during eclipse (or night) times and lower the total mass of the required solar power units.

\section{Crew Radiation Protection:}

According to Cucinotta ${ }^{14}$, liquid hydrogen shielding is the only currently feasible method of protecting astronauts from Galactic Cosmic Radiation (GCR). His models suggest that $10 \mathrm{~g} / \mathrm{cm}^{2}$ of hydrogen is marginally acceptable for a 500 day trip to Mars (excluding the time stayed on the planet). Ignoring the fact that this doesn't include the thickness needed to stop secondary radiation particles generated by the hydrogen containment (i.e. the tank), the thickness for $20 \mathrm{~g} / \mathrm{cm}^{2}$ is just less than 3 meters when the hydrogen is stored at 1 atmosphere. While the thickness can be varied by roughly $10 \%$ in either direction by changing the storage pressure, this still would place the diameter of even a long, slender $4 \mathrm{~m}$ diameter crew quarters over the $10 \mathrm{~m}$ diameter threshold that even SLS could lift. The mass of such a system would easily be on the order of hundreds of metric tons, the entire lift capability of SLS. The most efficient way to launch and load such a system is to load it on orbit, probably coexisting with a depot. Launching the transport stage several years in advance along with periodically supplying water to the PPLS would allow the fill up of the hydrogen shield over that time. 


\section{Development Path}

Minimal cryogenic storage durations for exploration beyond LEO will exceed our current capabilities by two or more orders of magnitude. This will require development and validation of a new suite of technologies, from large scale insulation systems to microgravity fluid control to active thermal control of cryogens for extended duration zero boil off. NASA is planning a Cryogenic Propellant Storage and Transfer (CPST) orbital demonstration in the 2017 timeframe that will prove many of the systems needed for long duration cryogenic storage in LEO, including active and passive thermal control, propellant acquisition and gauging, and cryogenic transfer between storage vessels. This mission should help push the current state of the art in long term cryogenic storage and handling to the TRL 7 level. But the scope of this mission is conservative and has limited relevance to a sustainable propellant depot.

There are several critical technologies at lower TRL available for propellant depots that can provide much greater capability and commercial viability. These include high capacity cryogenic refrigeration, low temperature spacecraft systems, and long life electrolysis units. Because propellant depots are such an enabling capability, these long term technology options should be investigated now to provide a path to the future. All three have been identified on the NASA Office of Chief Technologist (OCT) Roadmaps as needing more development ${ }^{15}$.

Several possible refrigeration cycles exist that can be used to liquefy the gaseous product from the electrolyzer. The leading candidate is a closed cycle Brayton refrigeration system that is designed to remove heat from the product stream in a series of stages, finalizing in a cold stage temperature a few degrees colder than the desired storage temperature. According to this method, after the hydrogen product stream exits the electrolysis unit, the hydrogen is pre-cooled in a radiator passively to a desired temperature. Next, a series of heat exchangers further cools the gas using a multi-stage Brayton refrigerator down to a temperature below $40 \mathrm{~K}$, after whcih a JouleThompson (JT) valve expands the gas isenthalpicaly into a two phase fluid. The refrigerator then provides the additional cooling necessary to liquefy the remaining saturated vapor in an expanded metal foam condensing heat exchanger prior to being delivered to the customer spacecraft as a single phase fluid. Since all the necessary refrigeration equipment will be on the depot, it can provide all the necessary refrigeration to the customer's spacecraft for full propellant conditioning during the entire time the two are docked. This enables a reduction of the customer's spacecraft mas.

The PPLS will use an integrated thermal insulation system designed to minimize parasitic loads on the refrigerator and will use a "heat stationing" approach in which warm components are located together at one end of the spacecraft and the progressively colder stages are positioned to take maximum advantage of the shielding (and active cooling) of the higher temperature stations. All penetrations between stations will use low conductivity supports and feedthrus where possible. Deployed solar shields may be used to protect against radiation, or the large solar arrays can be designed to fill that role. Since the PPLS is launched dry and refrigeration is not activated until orbital insertion, there is no need for hybrid insulation to protect systems during ground processing or ascent phases of the mission.

The prposed electrolysis unit consists of an electrolysis stack, probably a proton exchange membrane system, a gaseous purification process to ensure the product is ready for liquefaction, and the associated fluids and electrical controls components. The process is well understood and the engineering is mature. The electrolysis unit on the International Space Station (ISS) has displayed good reliability and the US Navy has extensive experience using larger electrolysis units for submarines; either of these systems could searve as a sound basis for in-space development. An advantage of the US Navy electrolysis development is high output pressures can be achieved for minimal power increase. Oxygen pressures up to $20 \mathrm{MPa}$ and hydrogen pressure up to $5.2 \mathrm{MPa}$ have been demonstrated. This minimizes the liquefaction energy required. Higher capacity space rated units with high outlet pressure are needed.

\section{Proposed Demonstration}

This paper serves as a pre-proposal to establish interest in NASA Advanced Exporation System (AES) funding for 2015-2017 to demonstrate the entire thermodynamic process of the PPLS in a relevant environment. Such a demonstration should serve to eliminate much of the technical risk associated with the proposed process and would take place on the International Space Station. Launch costs would need to be provided by another program, but AES funding would be sufficient to develop the prototype hardware, test it in a thermal vacuum chamber, prepare the payload for flight, and perform the in-space equipment checkout and testing. The ISS is an ideal location to perform this test and its assets would be necessary for the demonstration. The ISS already provides photovoltaic (PV) power similar to the proposed PPLS, therefore the deomonstration hardware would use that source of electrical 
power. The ISS also has a water supply source that could be used to simulate the WSM as well as radiators and ammonia cooling loops to reject the waste heat of the refrigeration cycle. An electrolysis unit is present on board that provides gaseous hydrogen to a Sabatier reactor, which, in turn, outputs water and gaseous methane. Both the methane and hydrogen feeds will be utilized in the cycle, simulating the PLSS hydrogen production. Finally, the ISS has the necessary data acquisition (DAQ) and communication systems for running the experiment and collecting and distributing the data.

AES funding will be used primarily to develop the experiment package that will include interfaces with the ISS power, data, cooling and $\mathrm{GH}_{2}$ sources, the $\mathrm{GH}_{2}$ liquefaction heat exchangers and J-T valves, the cryogenic refrigerator $(20 \mathrm{~W}$ at $20 \mathrm{~K}$ and $40 \mathrm{~W}$ at $90 \mathrm{~K}$ ), the cryogenic fluid management payload (mixing pump, spray bar, liquid acquisition device), a small ( $<200$ liter) liquid hydrogen tank, and the passive thermal control systems. The demonstration project will be a collaboration between multiple NASA centers, including Kennedy Space Center (KSC), Johnson Space Center (JSC), Glenn Research Center (GRC), and Goddard Space Flight Center (GSFC). As envisioned, $\mathrm{KSC}$ will responsible for the design and fabrication of the experiment pallet, the passive thermal control system, the fluid components, and the pallet integration and systems engineering; JSC will be responsible for integration of the pallet to the ISS, the command and control system, and the orbital test operations; GRC will be responsible for design, fabrication and integrating of the CFM components into the storage tank and the system modeling; and GSFC will be responsible for the development of the $20 \mathrm{~W}$ at $20 \mathrm{~K}$ cryogenic refrigerator. Figure 3 shows the conceptual refrigeration cycle for the proposed ISS experiment.

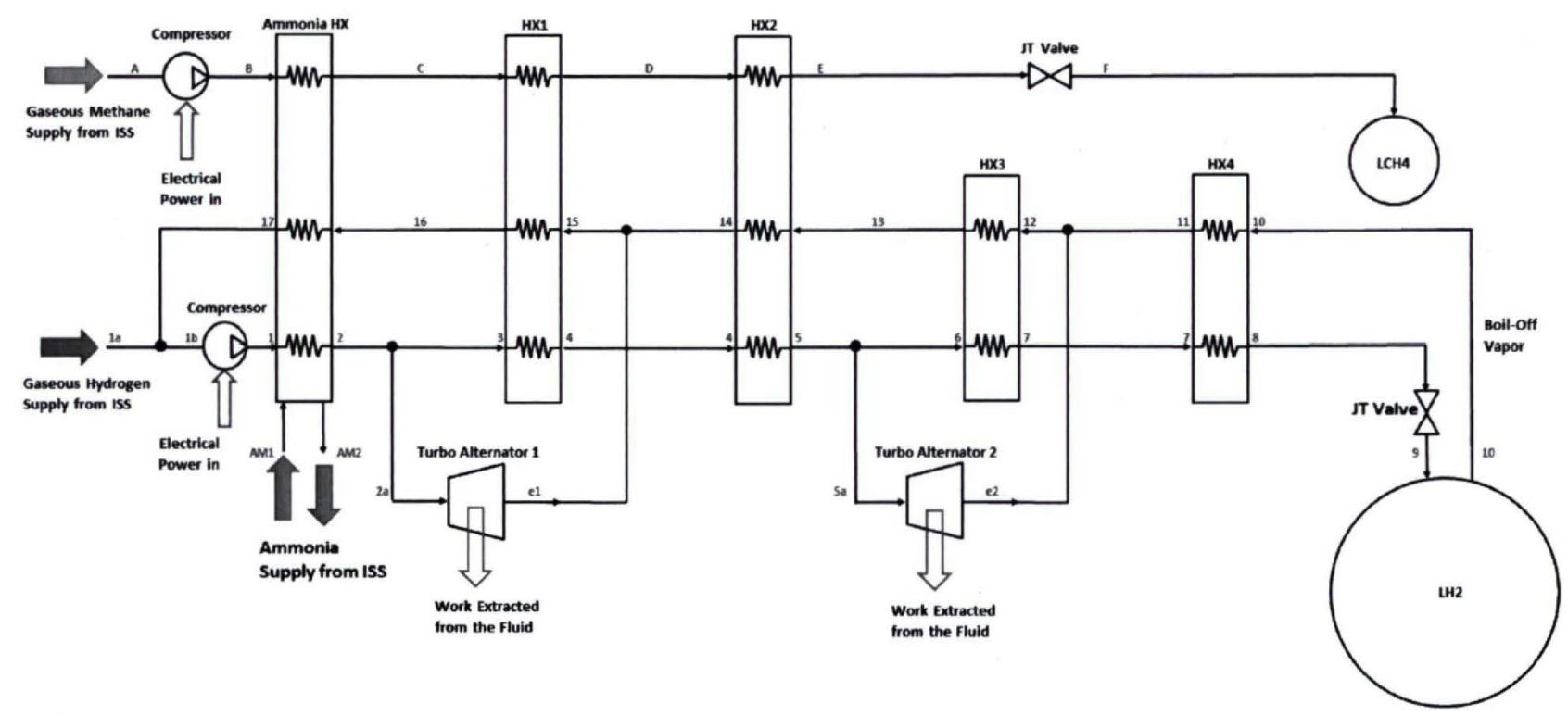

Figure 3: Proposed ISS Experiment Refrigeration/Liquefaction Cycle

\section{Deployment Path}

Although this article focuses on a NASA development path, given the current state of the NASA budget, it may be more realistic to assume that the first such cryogenic depot will be developed by commercial space companies, similar to the planned satellite re-servicing capability planned by MDA/IntelSat. As a result, alternative development paths to the one just described for near-term propellant depots should be investigated. These paths would need to take into account the commercial viability of providing consumables sales in space so that a solid business model can be developed. Terrestrial analogs include private companies investing hundreds of millions of dollars in industrial gas plants for consumables sales to customers, with the critical distinction being that in this case the plant is a propellant production satellite and the customers are owners/operators of launch vehicle upper stages that need refueling. 


\section{Conclusion}

NASA should pursue a LEO PPLS to enable high energy science missions and human interplanetary travel. This depot should then be moved to L1 if lunar In-Situ Resource Utilization (ISRU) enables the production of lunar water to be used in a cheaper manner.

\section{References}

2 "Can We Get to Mars?" (With Cornelius Ryan) Collier's, Vol. 133, No. 13, Apr.30, 1954, pp. 22-29

${ }^{3}$ Goff J., Kutter B., Zegler F., Bienhoff D., Chandler F., Marchetta J., "Realistic Near-Term Propelant Depots: Implementation of a Critical Spacefaring Capability," AIAA-2009-6756; proceedings of the AIAA SPACE 2009 Conference and Exposition, Pasadena, CA, Sep. 14-17, 2009

4 "Review of U.S. Human Space Flight Plans Committee, SEEKING A HUMAN SPACEFLIGHT PROGRAM WORTHY OF A GREAT NATION"; http://legislative.nasa.gov/396093main_HSF Cmte FinalReport.pdf

${ }^{5}$ Arney, D., Wilhite A., "Orbital Propellant Depots Enabling Lunar Architectures Without Heavy-Lift Launch Vehicles", J. Spacecraft and Rockets, Vol. 47, No. 2, March-April 2010. Pg. 353 - 360.

${ }^{6}$ Air Force Space Command, Range Safety User Requirements Manual (91-710) volume 3: Launch Vehicles, Payloads, and Ground Support Systems Requirements, 2004.

7 Clark, K., Magner, T., et. al., Jupiter Europa Orbiter Mission Study 2008: Final Report, 30 Jan 2009. http://opfm.jpl.nasa.gov/files/JEO-Rpt_Public-Release_090203.pdf

${ }^{8}$ McClesky, C.M., Space Shuttle Operations and Infrastructure - A Systems Analysis of Design Root Causes and Effects, NASA/TP 2005211519 ; NASA KSC

${ }^{9}$ Nast, T, Frank, D, and Burns, K, "Cryogenic propellant Boil Off Reduction Approaches," AIAA 2011-806, proceedings of 49th AIAA Aerospace Sciences Meeting, Jan 4-7, 2011, Orlando FL.

${ }^{10}$ Bock, E. H., Fisher, J. G., "In-Space Propellant Processing Using Water Delivered as Shuttle Contingency Payload," AIAA 78-941, proceedings of the $14^{\text {th }}$ AIAA/SAE Joint Propulsion Confrence, July 25-27, 1978, Las Vegas, NV.

${ }^{11}$ Moran, M., "Conceptual Study Of On Orbit Production Of Cryogenic Propellants By Water Electrolysis," AIAA 91-1844, proceedings of the $27^{\text {th }}$ AIAA/SAE/ASME/ASEE Joint Propulsion Conference, June 24-26, 1991, Sacramento, CA.

${ }^{12}$ Boeing, NASA MSFC, Sverdrup Technologies, "Space Solar Power and Platform Technologies for In-Space Propellant Depots," NAS8-99140, Mod. 2, Task 3.

${ }^{13}$ Notardonato, W., "Active Control of Cryogenic Propellants in Space," proceedings of the AIAA SPACE 2011 Conference and Exposition, Long Beach, CA, Sep. 27-29, 2011

${ }^{14}$ Cucinotta, F.A., Kim, M-H.Y., and Ren, L., "Evaluating shielding effectiveness for reducing space radiation cancer risks," Radiation Measurements, vol. 41, 2006, pg. 1173-1185.

${ }^{15}$ NASA Office of Chief Technologist website, http://www.nasa.gov/offices/oct/home/roadmaps/index.html 